\title{
The MERTK/FLT3 inhibitor MRX-2843 overcomes resistance-conferring FLT3 mutations in acute myeloid leukemia
}

\author{
Katherine A. Minson, ${ }^{1}$ Catherine C. Smith, ${ }^{2}$ Deborah DeRyckere, ${ }^{1,3}$ Clara Libbrecht, ${ }^{4}$ \\ Alisa B. Lee-Sherick, ${ }^{4}$ Madeline G. Huey, ${ }^{1}$ Elisabeth A. Lasater, ${ }^{2}$ Gregory D. Kirkpatrick, ${ }^{4}$ \\ Michael A. Stashko, ${ }^{5}$ Weihe Zhang, ${ }^{5}$ Craig T. Jordan, ${ }^{6}$ Dmitri Kireev, ${ }^{5}$ Xiaodong Wang, ${ }^{5}$ \\ Stephen V. Frye, ${ }^{5,7}$ H. Shelton Earp, ${ }^{7,8}$ Neil P. Shah, ${ }^{2}$ and Douglas K. Graham ${ }^{1,3}$
}

'Aflac Cancer Center of Children's Healthcare of Atlanta and Emory University Department of Pediatrics, Atlanta, Georgia, USA. ${ }^{2}$ UCSF, Department of Medicine, San Francisco, California, USA. ${ }^{3}$ Winship Cancer Institute, Emory University, Atlanta, GA, USA. ${ }^{4}$ University of Colorado, Department of Pediatrics, Aurora, Colorado, USA. ${ }^{5}$ University of North Carolina at Chapel Hill, Eshelman School of Pharmacy, Chapel Hill, North Carolina, USA. ${ }^{6}$ University of Colorado, Department of Medicine, Aurora, Colorado, USA. 'UNC Lineberger Comprehensive Cancer Center, University of North Carolina at Chapel Hill, Chapel Hill, North Carolina, USA. ${ }^{8}$ University of North Carolina at Chapel Hill, Department of Medicine, Chapel Hill, North Carolina, USA.

FMS-like tyrosine kinase 3-targeted (FLT3-targeted) therapies have shown initial promise for the treatment of acute myeloid leukemia (AML) expressing FLT3-activating mutations; however, resistance emerges rapidly. Furthermore, limited options exist for the treatment of FLT3independent $A M L$, demonstrating the need for novel therapies that reduce toxicity and improve survival. MERTK receptor tyrosine kinase is overexpressed in $80 \%$ to $90 \%$ of AMLs and contributes to leukemogenesis. Here, we describe MRX-2843, a type 1 small-molecule tyrosine kinase inhibitor that abrogates activation of both MERTK and FLT3 and their downstream effectors. MRX-2843 treatment induces apoptosis and inhibits colony formation in AML cell lines and primary patient samples expressing MERTK and/or FLT3-ITD, with a wide therapeutic window compared with that of normal human cord blood cells. In murine orthotopic xenograft models, once-daily oral therapy prolonged survival 2- to 3-fold over that of vehicle-treated controls. Additionally, MRX-2843 retained activity against quizartinib-resistant FLT3-ITD-mutant proteins with clinically relevant alterations at the D835 or F691 loci and prolonged survival in xenograft models of quizartinibresistant AML. Together, these observations validate MRX-2843 as a translational agent and support its clinical development for the treatment of AML.

Conflict of interest: D.K. Graham, D. DeRyckere, and H.S. Earp have filed patents on targeting of the MERTK tyrosine kinase as cancer therapy. D. Kireev, X. Wang, W. Zhang, and S.V. Frye have filed patents on MRX-2843 Additionally, D. DeRyckere, D. Kireev, X. Wang, S.V. Frye, H.S. Earp, and D.K. Graham hold stock in Meryx Inc. (a company developing novel therapeutics against MERTK).

Submitted: November 20, 2015 Accepted: February 17, 2016 Published: March 17, 2016

Reference information: JCI Insight. 2016;1(3):e85630. doi:10.1172/jici.insight.85630.

\section{Introduction}

Acute myeloid leukemia (AML) continues to have a poor prognosis, despite therapeutic advancements over the past several decades, as survival rates are only $60 \%$ to $70 \%$ in pediatric patients and less than $50 \%$ in adult patients $(1,2)$. Older patients have progressively worse outcomes that are at least partly due to an inability to tolerate aggressive cytotoxic chemotherapy (3). In pediatric patients, these therapies can lead to devastating long-term side effects, including growth hormone deficiency, neurocognitive abnormalities, and infertility (4). These observations demonstrate the need for novel, better-tolerated therapies in AML patients and have led to the development of a number of targeted agents over the past decade. For instance, numerous small-molecule inhibitors targeting the often-mutated FMS-like tyrosine kinase 3 (FLT3) are currently in clinical trials.

Internal tandem duplication (ITD) mutations that confer constitutive kinase activation in FLT3 (FLT3ITD) are observed in $20 \%$ to $30 \%$ of adults and $10 \%$ to $15 \%$ of children with AML and are associated with poor prognosis $(5,6)$. Although many patients have a good initial response to FLT3 inhibition, sustained responses have been less successful. In a phase II study of quizartinib monotherapy in patients with relapsed or refractory FLT3-ITD AML, a 44\% composite complete remission was achieved; however, development of resistance was rapid, and the median duration of response was only approximately 11 
A

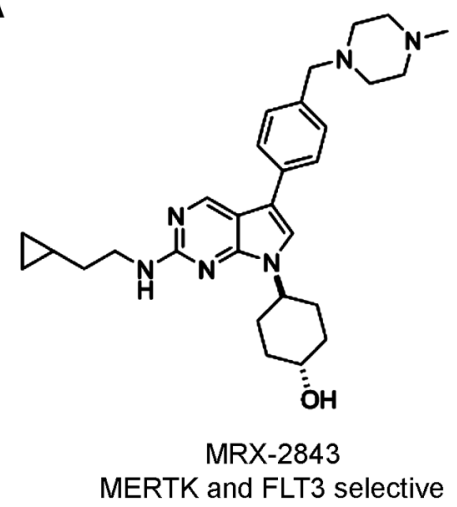

B

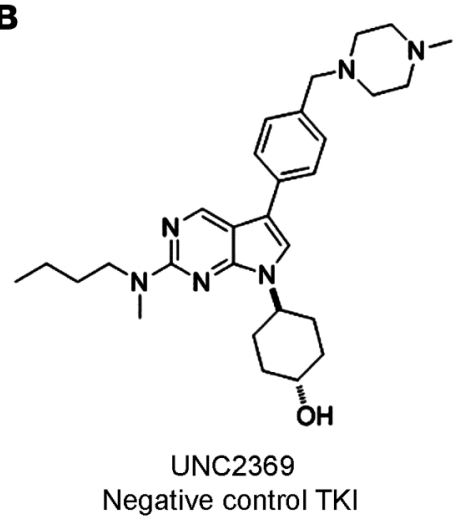

Figure 1. MRX-2843 is a dual MERTK and FLT3 TKI. (A) Chemical structure of MRX-2843, with an enzymatic IC ${ }_{50}$ of 1.3 nM for MERTK and 0.64 nM for FLT3 (B) Chemical structure of UNC2369, which lacks significant activity against MERTK (enzymatic IC of $_{50} 490 \mathrm{nM}$ ) or FLT3 (enzymatic IC $\mathrm{I}_{50}$ of $360 \mathrm{nM}$ ) and was used as a negative control in these studies. FLT3, FMS-like tyrosine kinase 3; TKI, tyrosine kinase inhibitor.

weeks (7). Subsequent studies utilizing saturation mutagenesis in cell culture assays identified 3 residues in the FLT3 kinase domain that conferred resistance to quizartinib -2 within the kinase activation loop (D835 and Y842) and 1 at a gatekeeper position (F691). Mutations at 2 of these loci - D835 and F691 - were subsequently identified in patients who relapsed while on quizartinib monotherapy, confirming the clinical relevance of these loci (8). In addition, the acquisition of mutations at D835 has also been reported after treatment with sorafenib, a first-generation FLT3 inhibitor (9). Although tyrosine kinase inhibitors (TKIs) that retain activity against either the D835Y activation loop mutation (crenolanib) or the F691L gatekeeper mutation (ponatinib) have been developed, inhibitors that retain equal activity against both mutations have not been reported $(10,11)$. While these previous studies have validated FLT3 inhibition as a therapeutic strategy for the treatment of patients with AML, more effective agents targeting FLT3-ITD are needed.

Similarly, therapeutic agents directed against novel targets may augment the response to FLT3 inhibitors and are also needed for treatment of AMLs without a FLT3-ITD mutation. MERTK is a receptor tyrosine kinase that is ectopically expressed in the majority of acute leukemias, and increasing evidence suggests a role for MERTK in multiple solid tumors (12-18). In AML, MERTK is overexpressed on more than $80 \%$ of pediatric and adult patient samples relative to normal bone marrow precursor cells $(13,14)$. Importantly, shRNA-mediated inhibition of MERTK in AML led to decreased signaling through prosurvival pathways, inhibited colony formation, induced apoptosis, and prolonged survival in murine models (13), indicating that MERTK inhibition has therapeutic potential.

We have previously described UNC1666, a first-generation small-molecule inhibitor of both MERTK and FLT3 with potent antileukemia activity (19). Unfortunately, this compound has a limited half-life in animal models and is therefore not appropriate for clinical development. Here, we report preclinical testing of MRX-2843, an orally available small-molecule inhibitor of both MERTK and FLT3 (20). We demonstrate potent antileukemia activity mediated by MRX-2843 in MERTK-dependent and FLT3-ITD models of AML. Moreover, MRX-2843 retains the ability to inhibit activation of both quizartinib-resistant FLT3ITD D835Y- and F691L-mutant proteins and mediates potent antileukemia activity when either of these clinically relevant FLT3 point mutations is present. Combined, these data form the basis for further exploration of the clinical utility of MRX-2843 in AML.

\section{Results}

MRX-2483 is a novel small-molecule inhibitor with selectivity for MERTK and FLT3. We have previously reported the synthesis and in vivo pharmacokinetic parameters of TKIs of MERTK, including MRX-2843 (Compound 12; ref. 20). MRX-2843 is an orally bioavailable ATP-competitive type 1 TKI with high potency against both MERTK and FLT3 in enzymatic assays (Figure 1A) and with selectivity for these kinases over the other members of the TAM-family, AXL and TYRO-3, and other relevant tyrosine kinases (Supplemental Table 1; supplemental material available online with this article; doi:10.1172/jci.insight.85630DS1). 
A

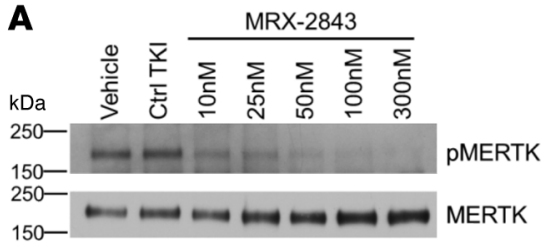

B

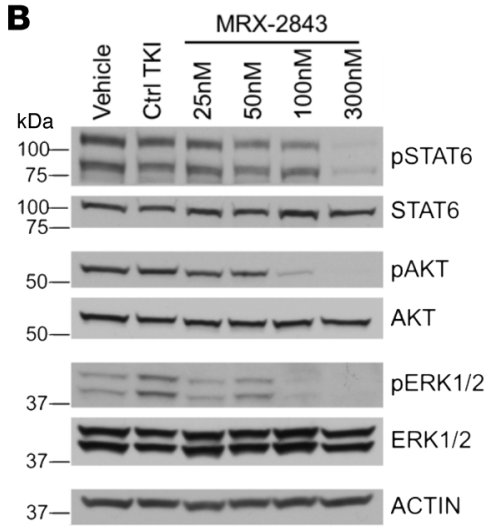

C

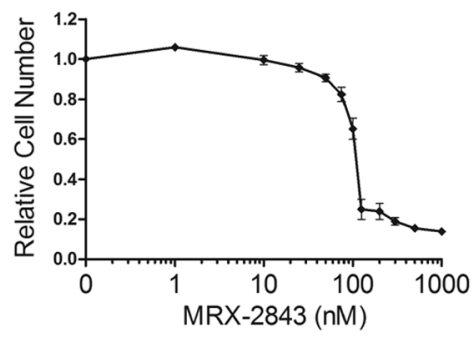

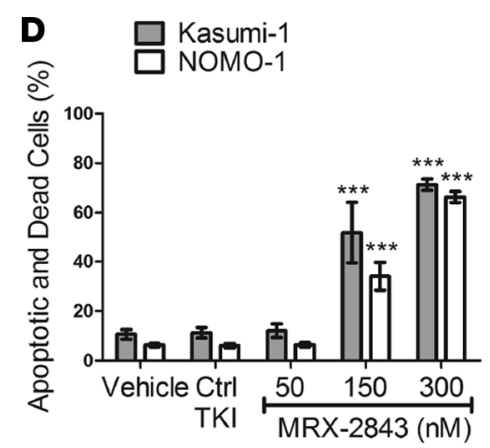

$\mathbf{F}$

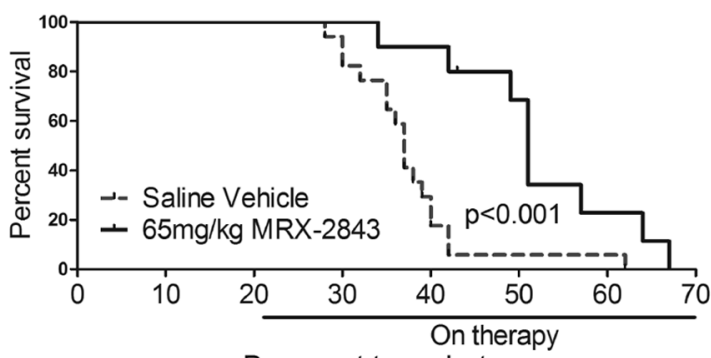

Figure 2. MRX-2843 inhibits MERTK activation and downstream signaling and has functional antitumor effects in MERTK ${ }^{+}$FLT3-WT cell culture and animal models. (A-D) Kasumi-1 and/or NOMO-1 AML cells were treated with the indicated concentrations of MRX-2843, vehicle (DMSO), or a nontargeting control TKI (Ctrl TKI). (A) After 1 hour, Kasumi-1 cells were treated with pervanadate phosphatase inhibitor for 10 minutes to stabilize $p$-MERTK, and cell lysates were prepared. MERTK protein was immunoprecipitated, and p-MERTK and total MERTK were detected by immunoblot analysis. (B) After 2 hours, lysates were prepared from Kasumi-1 cells without pervanadate treatment, and phosphorylated and total STAT6, AKT, and ERK1/2 proteins were detected by immunoblotting. Actin was used as a loading control. (C) After 48 hours, MTS reagent was added to cultures for an additional 2 hours, and absorbance was determined as an indicator of the number of viable cells. (D) After 72 hours, cells were stained with Yo-Pro-1 iodide and propidium iodide, and apoptotic and dead cells were detected by flow cytometry. ${ }^{* *} P<0.001$, by 1-way ANOVA. (E) Kasumi-1 or NOMO-1 cells were cultured in soft agar overlaid with media containing the indicated concentrations of MRX-2843, vehicle (DMSO), or control TKI. Colonies were stained and counted after 14 to 21 days. Results are shown relative to vehicle-treated controls. Mean values and standard errors were derived from 3 independent experiments. ${ }^{* *} P<0.01$ and ${ }^{* * *} P<$ 0.001 , by 1-way ANOVA. (F) Kaplan-Meier curve showing survival of NSGS mice with orthotopic NOMO-1 xenografts that were treated with MRX-2843 or vehicle (saline) beginning on day 21 after transplantation. Median survival was 51 days for MRX-2843-treated mice and 37 days for vehicle-treated control mice ( $n \geq 10$ per group). $P<0.001$, by log-rank test. AML, acute myeloid leukemia; Ctrl TKI, control tyrosine kinase inhibitor; FLT3, FMS-like tyrosine kinase 3; NSCS, NOD-SCID- $\gamma$ mice expressing Tg human cytokines; $p$, phosphorylated.

In contrast, UNC2369 (Compound 20; ref. 20) has a similar structure but much lower potency against MERTK and FLT3 and was used as a negative control TKI in these studies (Figure 1B). In mice, MRX2843 is $78 \%$ orally bioavailable at a dose of $3 \mathrm{mg} / \mathrm{kg}$ with a $\mathrm{C}_{\max }$ of $1.3 \mu \mathrm{M}$ and a $t_{1 / 2}$ of 4.4 hours (20).

$M R X-2843$ inhibits MERTK activation and mediates functional antileukemia effects in MERTK-dependent AML models. Cell lines that express MERTK (19) and have no activating FLT3 mutations (FLT3-WT) were studied to assess the effects of MRX-2843 on MERTK activity and leukemogenic functions. In the Kasumi-1 cell line, treatment with MRX-2843 resulted in dose-dependent inhibition of MERTK phosphorylation (Figure 2A). Decreased phosphorylation was evident at concentrations as low as $10 \mathrm{nM}$, with near-complete abrogation of MERTK activation at 100 to $300 \mathrm{nM}$. Similarly, treatment of Kasumi-1 cells with MRX-2843 mediated inhibition of downstream signaling through pathways important for tumor cell survival and proliferation. Phosphorylation inhibition of ERK1/2, AKT, and STAT6, signaling proteins known to be regulated downstream of MERTK (13), was inhibited in a dose-dependent fashion similar to the dose (25-300 nM) required for MERTK phosphorylation inhibition (Figure 2B). In contrast, ERK1/2, AKT, and STAT6 phosphorylation was not affected by treatment with the control TKI (Figure 2, A and B).

To determine the effect of treatment with MRX-2843 on clonal expansion, Kasumi-1 cells were cultured in the presence of MRX-2843, and cell numbers relative to vehicle-treated cultures were determined. 
A

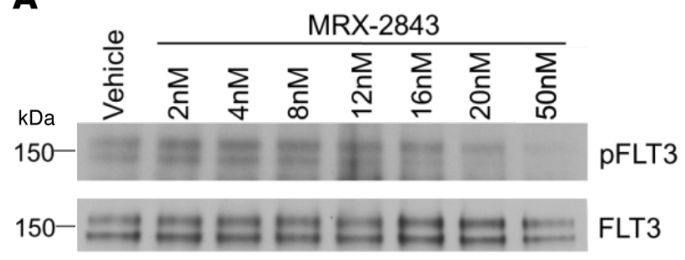

B

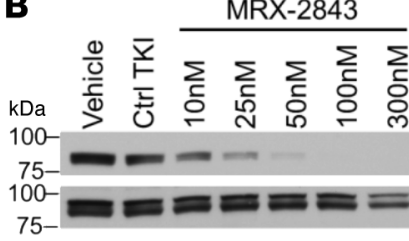

pSTAT5
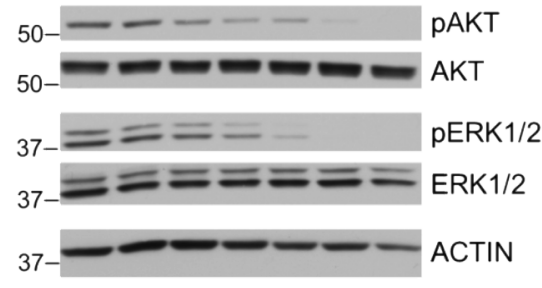

C

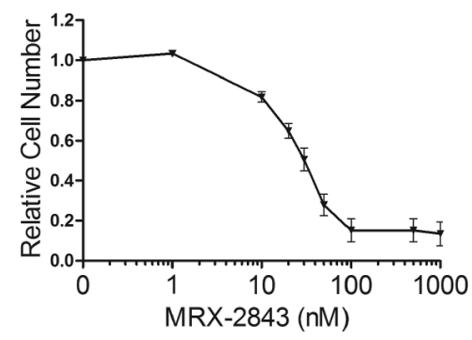

D

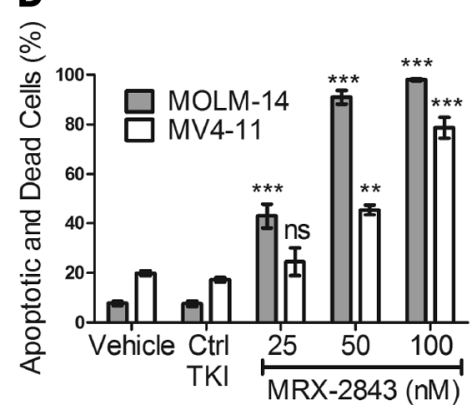

E

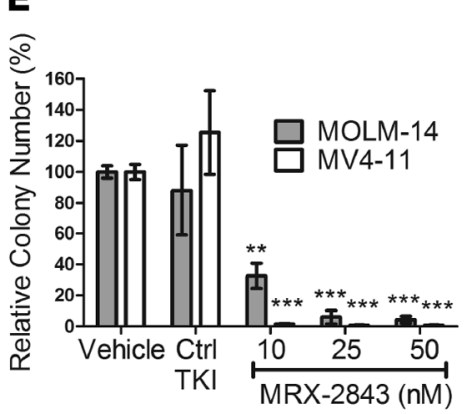

$\mathbf{F}$

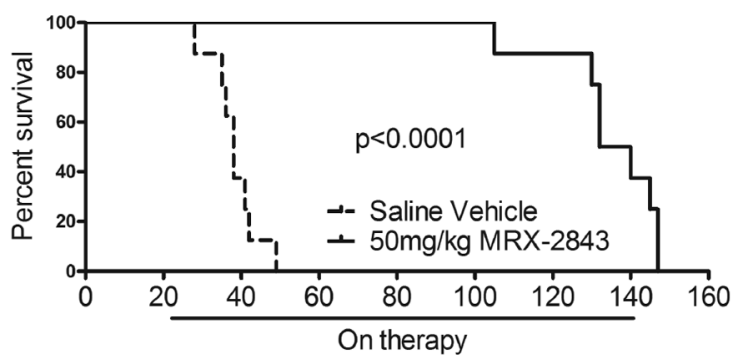

Days post-transplant

Figure 3. MRX-2843 inhibits FLT3 activation and downstream signaling and has functional antitumor effects in MERTK ${ }^{\text {neg }}$ FLT3-ITD cell lines. (A-D) MOLM-14 (MERTK $\left.{ }^{\text {neg }} F L T 3-I T D\right)$ and MV4-11 (MERTK ${ }^{\text {dim } F L T 3-I T D) ~ A M L ~ c e l l s ~ w e r e ~ t r e a t e d ~ w i t h ~ t h e ~ i n d i c a t e d ~ d o s e s ~ o f ~ M R X-2843, ~ v e h i c l e ~}$ (DMSO), or a nontargeting control TKI. (A) After 1 hour, MOLM-14 cells were treated with pervanadate phosphatase inhibitor for 3 minutes and lysates prepared. FLT3 protein was immunoprecipitated, and phosphorylated and total FLT3 were detected by immunoblot analysis. (B) Lysates were prepared from MOLM-14 cells without pervanadate treatment, and phosphorylated and total STAT5, AKT, and ERK1/2 proteins were detected by immunoblot analysis. Actin was used as a loading control. (C) After 48 hours, MTS reagent was added to cultures for an additional 2 hours, and absorbance was determined as an indicator of viable cell numbers. (D) After 72 hours of drug treatment, cells were stained with YoPro-1 iodide and propidium iodide dyes, and apoptotic and dead cells were detected by flow cytometry. ${ }^{* *} P<0.01$ and ${ }^{* * *} P<0.001$, by 1 -way ANOVA. (E) MOLM-14 or MV4-11 cells were cultured in soft agar overlaid with media containing the indicated concentrations of MRX-2843, vehicle (DMSO), or control TKI. Colonies were stained and counted after 14 to 21 days. Results are shown relative to vehicle-treated controls. Mean values and standard errors were derived from 3 independent experiments. ${ }^{* *} P<0.01$ and ${ }^{* * *} P<0.001$, by 1-way ANOVA. (F) Kaplan-Meier curve showing survival of NSG mice with orthotopic MOLM-14 xenografts that were treated with MRX-2843 or vehicle (saline) beginning on day 21 after transplantation. Median survival for MRX-2843-treated mice was 126 days compared with 38 days for vehicle-treated controls ( $n=8$ per group). $P<0.001$, by log-rank test. AML, acute myeloid leukemia; Ctrl TKI, control tyrosine kinase inhibitor; FLT3, FMS-like tyrosine kinase 3; ITD, internal tandem duplication; NSG, NOD-SCID- $\gamma$; p, phosphorylated.

MRX-2843 treatment resulted in a decrease in relative cell numbers, with an $\mathrm{IC}_{50}$ of $143.5 \pm 14.1 \mathrm{nM}$, indicating that MRX-2843 significantly inhibited tumor cell proliferation and/or survival (Figure 2C).

To more directly assess the impact of treatment with MRX-2843 on cell survival, AML cells were treated with MRX-2843, the control TKI, or vehicle (DMSO), and the percentage of apoptotic and dead cells was determined by flow cytometry. Both Kasumi-1 and NOMO-1 cells exhibited a statistically significant induction of cell death that was dose dependent and correlated with the degree of inhibition of MERTK phosphorylation and downstream signaling in Kasumi- 1 cells. At doses of $150 \mathrm{nM}$ and $300 \mathrm{nM}$ in the Kasumi-1 line, the fraction of apoptotic and dead cells was $51.9 \% \pm 12.4 \%$ and $71.3 \% \pm 2.3 \%$, respectively, compared with $10.6 \% \pm 2.0 \%$ in the vehicle-treated cultures $(P<0.001$, Figure $2 \mathrm{D})$. Similarly, there were $34.1 \% \pm 5.6 \%$ and $67.1 \% \pm 2.7 \%$ apoptotic and dead cells in NOMO- 1 cultures treated with $150 \mathrm{nM}$ or $300 \mathrm{nM}$ MRX-2843, respectively, compared with $6.8 \% \pm 0.7 \%$ in vehicle-treated cultures $(P<0.001$, Figure 2D). In contrast, treatment with control TKI had no significant effect on cell survival.

To further evaluate the functional effects of MRX-2843, colony-forming potential was assessed in soft agar cultures. Kasumi-1 or NOMO-1 cells were cultured in equal numbers in soft agar overlaid with medium containing MRX-2843, the control TKI, or vehicle for 14 days, and the colonies were counted. In both cell 
A

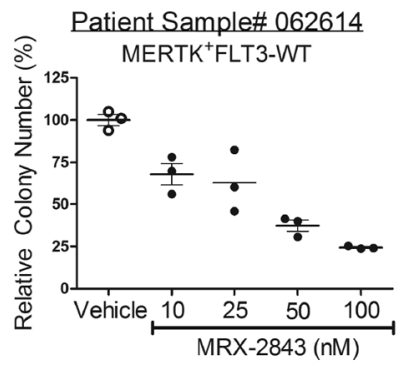

B

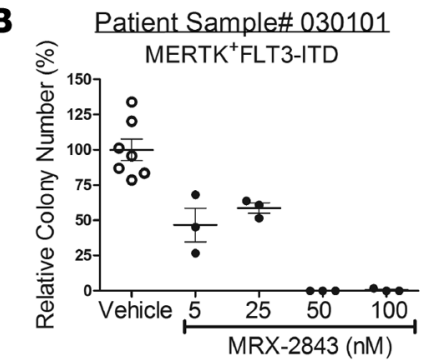

C

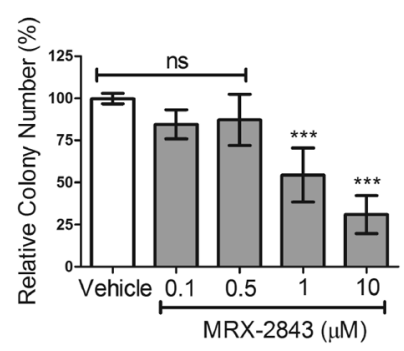

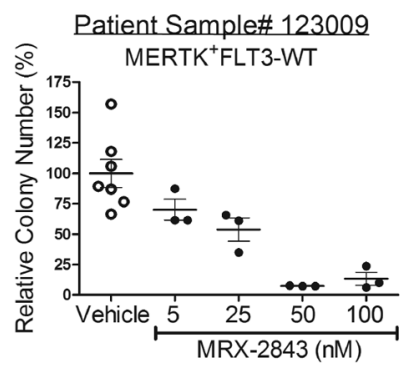

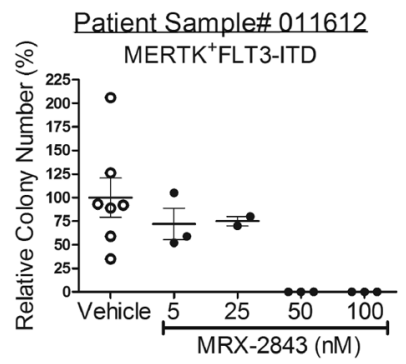

Figure 4. MRX-2843 inhibits colony formation in MERTK-expressing and FLT3-ITD primary AML patient samples. (A and B) Primary AML patient samples were cultured in methylcellulose medium with the indicated concentrations of MRX-2843 or vehicle (DMSO). Colonies were stained and counted after 10 days. Each sample was plated in triplicate, and mean values and standard errors are shown. (C) Human cord blood collected from healthy donors was cultured in methylcellulose, and colonies were counted as above. Mean values and standard errors were derived from 3 independent experiments and are relative to vehicle-treated controls. ${ }^{*} P<0.01$ and ${ }^{*}{ }^{*} P<0.001$, by 1 -way ANOVA. AML, acute myeloid leukemia; FLT3, FMS-like tyrosine kinase 3; ITD, internal tandem duplication.

lines, treatment with MRX-2843 significantly reduced colony numbers relative to those in the vehicle control in a dose-dependent manner (Figure 2E). Treatment with $50 \mathrm{nM}$ and $100 \mathrm{nM}$ MRX-2843 resulted in $62.3 \%$ $\pm 6.4 \%$ and $84.1 \% \pm 7.8 \%$ inhibition of colony formation, respectively, in Kasumi- 1 cultures $(P<0.01)$. Similarly, in NOMO-1 cultures, colony formation was inhibited by $54.8 \% \pm 18.1 \%$ in response to treatment with $100 \mathrm{nM}$ MRX-2843 $(P<0.001$, Figure 2E). Treatment with the control TKI had no significant effect.

To determine whether inhibition of oncogenic phenotypes in MERTK-expressing AML cells translates to animal models, orthotopic murine xenografts were established, and the effects of treatment with MRX-2843 on survival were determined. NOMO-1 cells were injected into the tail veins of NOD-SCID- $\gamma$ (NSG) mice expressing Tg human cytokines (NSGS), and $65 \mathrm{mg} / \mathrm{kg}$ MRX-2843 or an equivalent volume of vehicle (saline) was administered once daily by oral gavage starting 21 days after transplantation. Treatment with MRX-2843 significantly prolonged survival relative to that of vehicle-treated mice, with median survival increasing from 37 days to 51 days $(P<0.001$, Figure $2 \mathrm{~F})$.

Taken together, these data demonstrate inhibition of MERTK signaling, induction of cell death, and abrogation of oncogenic phenotypes in AML cells treated with MRX-2843. Moreover, MRX-2843 had therapeutic activity in a MERTK-dependent xenograft model, validating the use of MERTK inhibitors as a translational strategy for the treatment of AML.

MRX-2843 inhibits FLT3 activation and mediates functional antileukemic effects in FLT3-ITD AML models. FLT3ITD AML cell lines were used to assess the effects of treatment with MRX-2843 on FLT3-ITD activity and leukemogenic functions. The MOLM-14 cell line is FLT3-ITD heterozygous and does not express MERTK (MERT$\mathrm{K}^{\text {neg }}$ ); MV4-11 is FLT3-ITD homozygous and expresses only low levels of MERTK protein (MERTK ${ }^{\mathrm{dim}}$ ) (19). 
A Patient Sample\# 123009 MERTK ${ }^{+}$FLT3-WT

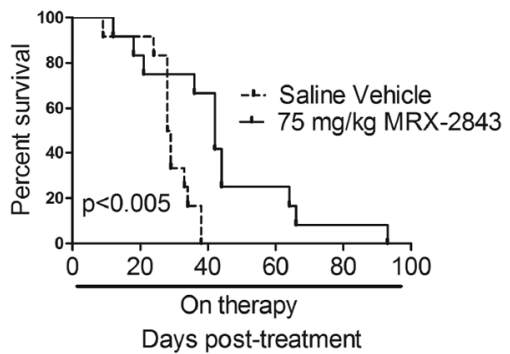

\section{B}

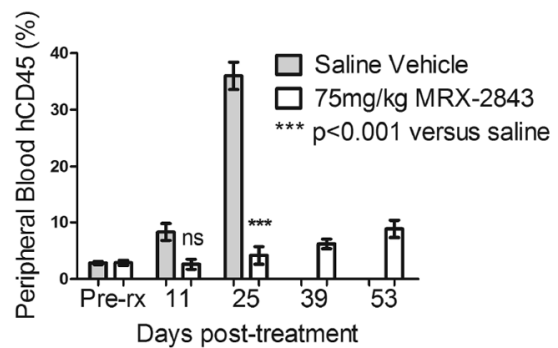

C

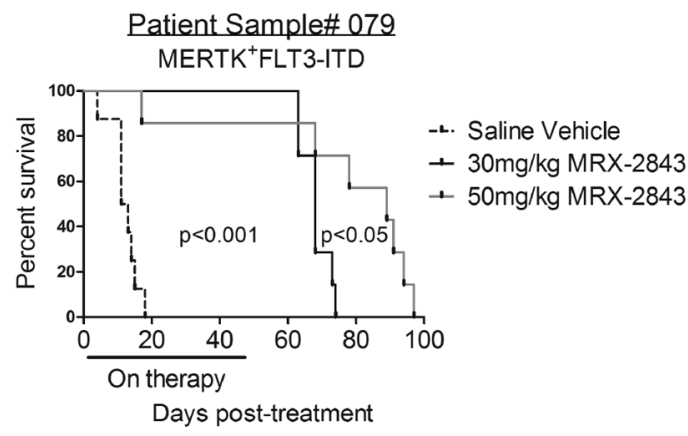

D

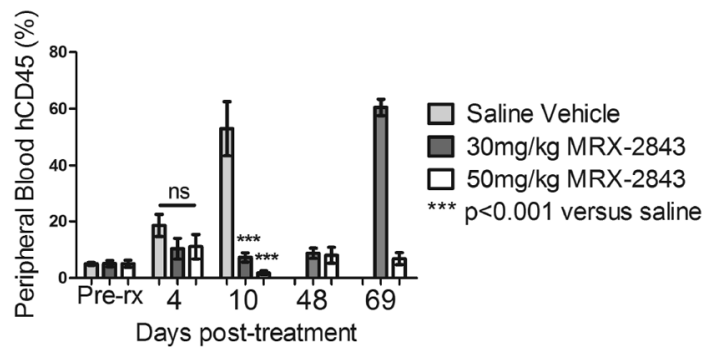

Figure 5. MRX-2843 prolongs survival in patient-derived xenograft models of AML. (A and C) Kaplan-Meier curves showing survival of NSCS mice xenografted with the indicated patient sample and treated with MRX-2843 or vehicle (saline). (B and D) Peripheral disease burden (percentage of hCD45+ peripheral blood mononuclear cells) was determined by flow cytometry 3 days prior to initiation of treatment (Pre-rx) and on the indicated days after treatment. Mean values and standard errors are shown. (A and B) Mice were started on therapy 53 days after transplantation with leukemia cells (day 0 ). Median survival of MRX-2843-treated mice after treatment was 42 days compared with 28.5 days for vehicle-treated controls ( $n=12$ per group). $P<0.005$, by log-rank test (A); ${ }^{* *} P<0.001$ versus saline, by 1-way ANOVA (B). (C and D) Mice were started on therapy 42 days after transplantation (day 0 ). Median survival of MRX-2843-treated mice was 68 days (30 mg/kg MRX-2843) and 89 days (50 mg/kg MRX-2843) after treatment compared with 12 days for vehicle-treated controls ( $n \geq 8$ per group). $P<0.001$ and $P<0.05$, by log-rank test (C); ${ }^{* *} P<0.001$ versus saline, by 1 -way ANOVA (D). AML, acute myeloid leukemia; FLT3, FMS-like tyrosine kinase 3; hCD45+, human CD45+; NSCS, NOD-SCID- $\gamma$ mice expressing Tg human cytokines. NOD-SCID- $\gamma$ mice expressing Tg human cytokines

In MOLM-14 cells, treatment with MRX-2843 inhibited phosphorylation of FLT3 and downstream signaling through STAT5, ERK1/2, and AKT (Figure 3, A and B). Activation of FLT3 and its signaling pathways was almost completely abrogated by treatment with $50 \mathrm{nM}$ MRX-2843, indicating somewhat higher cellular potency against FLT3 relative to MERTK. This finding was borne out in functional assays, with the 2 FLT3-dependent cell lines being more sensitive to MRX-2843 than the MERTK-dependent cell lines tested (Figure 2, C-E, and Figure 3, C-E). Treatment with MRX-2843 inhibited clonal expansion in MOLM-14 cultures, with an $\mathrm{IC}_{50}$ of $29.5 \pm 3.4 \mathrm{nM}$ (Figure 3C), approximately 4-fold lower than that in Kasumi-1 cells. Similarly, significant induction of apoptosis and inhibition of colony formation was observed at lower doses of MRX-2843 (Figure 3, D and E). In MOLM-14 cells, treatment with $50 \mathrm{nM}, \mathrm{MRX}-2843$ induced cell death in $91.0 \% \pm 2.7 \%$ cells $(P<0.001)$, whereas Kasumi-1 and NOMO-1 cells were not affected at this dose. MV4-11 cells were slightly less sensitive, with $45.6 \% \pm 4.2 \%$ apoptotic and dead cells after treatment with $50 \mathrm{nM} \mathrm{MRX}-2843(P<0.01)$ and 59.6\% $\pm 4.1 \%$ after treatment with $100 \mathrm{nM} \mathrm{MRX}-2843(P<0.001)$. Similarly, treatment with MRX-2843 inhibited colony formation in soft agar at concentrations as low as $10 \mathrm{nM}$, with near-complete abrogation of colonies in both cells lines in response to treatment with $25 \mathrm{nM}$ (Figure 3E). Interestingly, while MOLM-14 cells were more sensitive to MRX-2843 than MV4-11 cells in apoptosis assays, MV4-11 cells were more sensitive in soft agar cultures, where treatment with $10 \mathrm{nM} \mathrm{MRX-}$ 2843 resulted in a $98.4 \% \pm 0.4 \%$ reduction in colony formation relative to that in controls in the MV4-11 line compared with a $66.2 \% \pm 7.9 \%$ reduction in colony formation in MOLM-14 cultures.

To assess the effects of MRX-2843 treatment on FLT3-dependent leukemogenesis in vivo, orthotopic murine xenografts were generated by injecting MOLM-14 cells into the tail veins of NSG mice. Bone marrow engraftment was monitored by flow cytometry to determine the presence of human leukemia cells $\left(\mathrm{hCD} 45^{+}\right.$). Once-daily treatment with $50 \mathrm{mg} / \mathrm{kg} \mathrm{MRX}-2843$ or an equivalent volume of vehicle (saline) was initiated 21 days after transplantation, when mice had an average of $10.7 \% \pm 1.4 \%$ bone marrow blasts (data not shown), and therapy was continued until 140 days after transplantation. Mice treated with MRX2843 had significantly prolonged survival compared with that of mice treated with vehicle, with median 
A

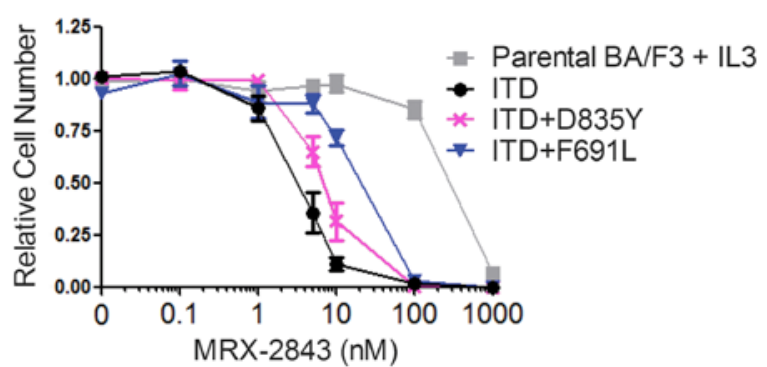

B

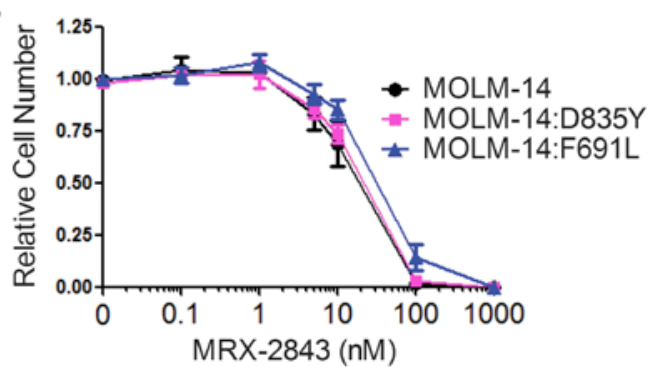

C

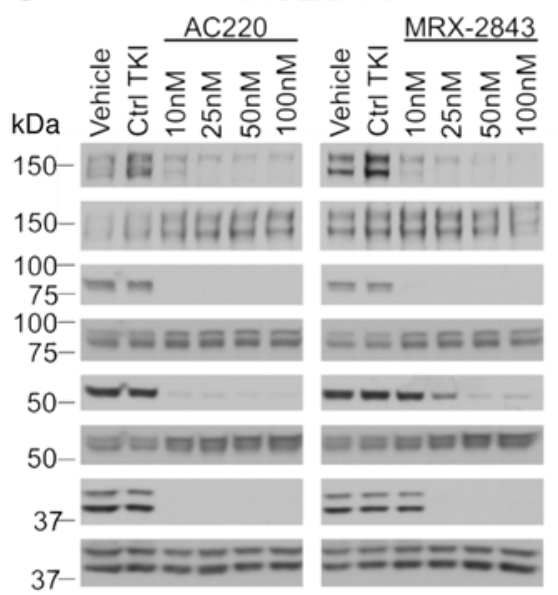

MOLM-14:D835Y

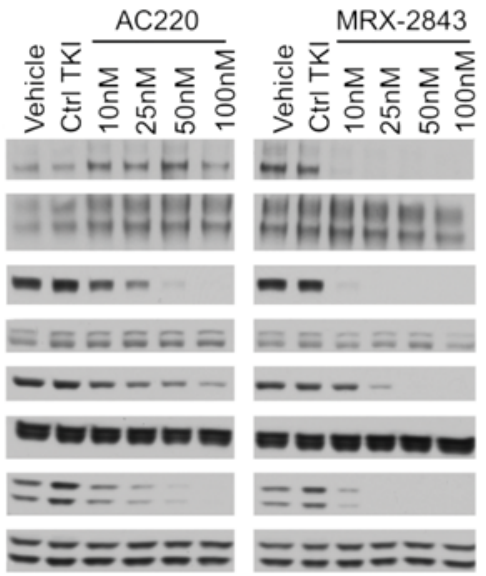

MOLM-14:F691L
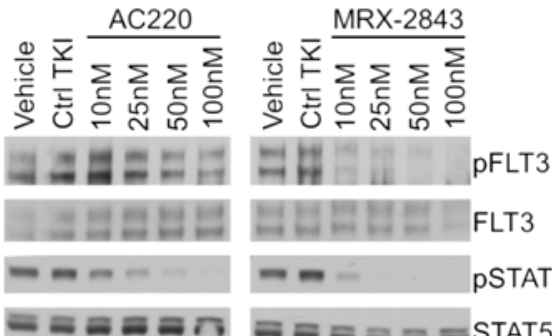

$=-\quad$ pSTAT5

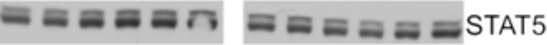

- - - - - -

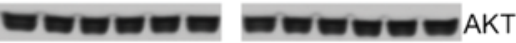

シニニニニニ シニニニ

シニニニニニ シニニニニニ ERK1/2

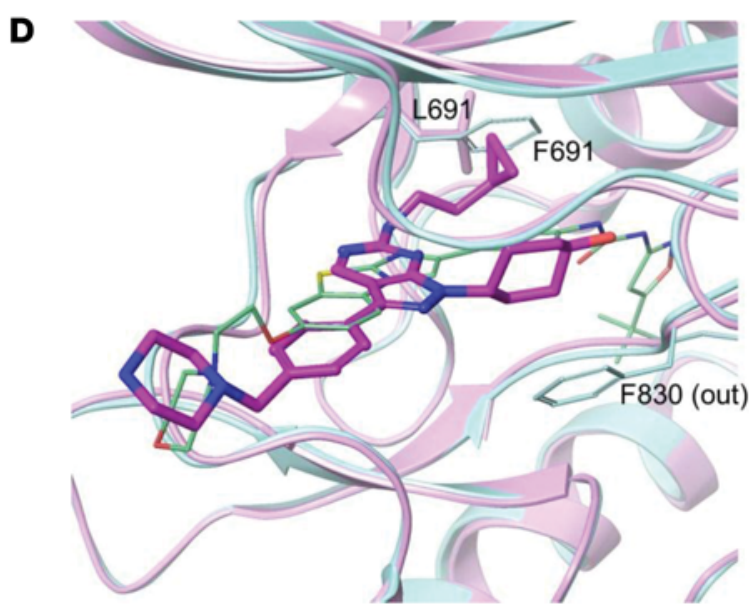

Figure 6. MRX-2843 retains activity against clinically relevant quizartinib-resistant FLT3 mutant proteins. (A) BA/F3 cells expressing FLT3-ITD or an FLT3-ITD mutant (D835Y or F691L) were cultured in the absence of IL-3 and treated with the indicated concentrations of MRX-2843. After 48 hours, CellTiter-Glo reagent was added to cultures, and absorbance was determined as an indicator of viable cell numbers relative to vehicle-treated controls. Cultures grown in the presence of IL-3 are shown for reference (Parental BA/F3 + IL-3). Each condition was assessed in triplicate, and mean values and standard errors were derived from 3 independent experiments. (B) WT MOLM-14 and derivative cell lines expressing a D835Y- or F691L-mutant FLT3-ITD were cultured in the presence of MRX-2843, and relative numbers of viable cells were determined as above. (C) MOLM-14 and derivative cell lines were cultured in the presence of MRX-2843, quizartinib (AC220), vehicle (DMSO), or a nontargeting control TKI for 1 hour, and signaling effectors downstream of FLT3 were detected by immunoblot analysis as described in Figure 3B. (D) Structural model of MRX-2843 (thick bars) and quizartinib (thin bars) bound to the FLT3 F691L mutant (magenta) and FLT3 WT (cyan). Positions of F691 (the gatekeeper) and F830 (part of the DFG-out motif) are indicated. Ctrl TKI, control tyrosine kinase inhibitor; FLT3, FMS-like tyrosine kinase 3; ITD, internal tandem duplication; p, phosphorylated.

survival of 136 days and 38 days, respectively $(P<0.0001$, Figure $3 F)$. Taken together, these data support the potential utility of MRX-2843 for treatment of FLT3-ITD AML.

MRX-2843 selectively inhibits colony formation in primary AML patient samples and prolongs survival in patient-derived xenograft models of AML. To determine whether MRX-2843 has activity against patient-de- 
A
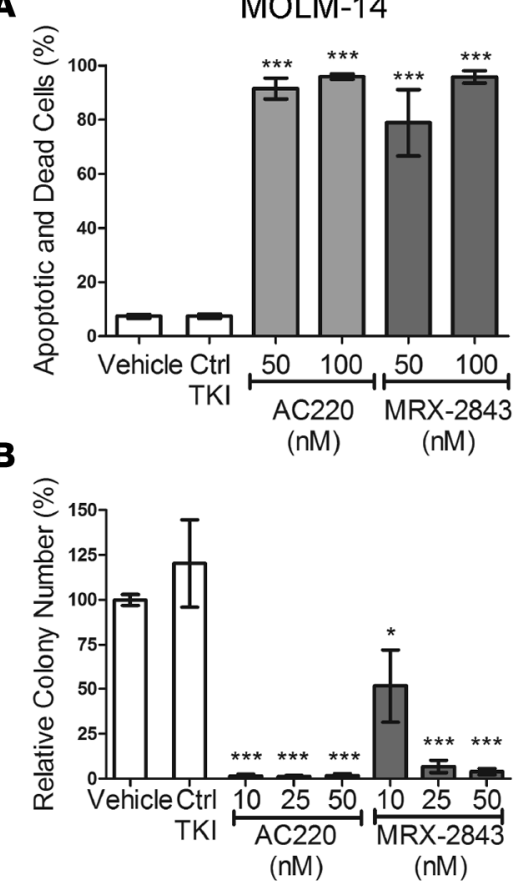

MOLM-14:D835Y
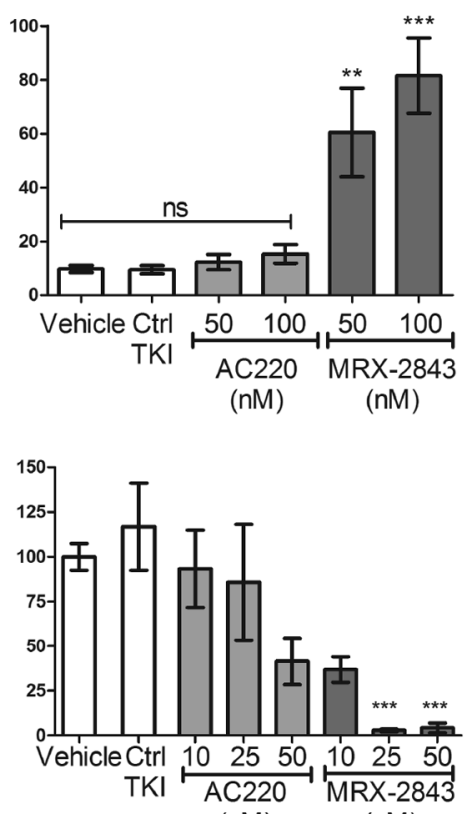

(nM)

(nM)

MOLM-14:F691L
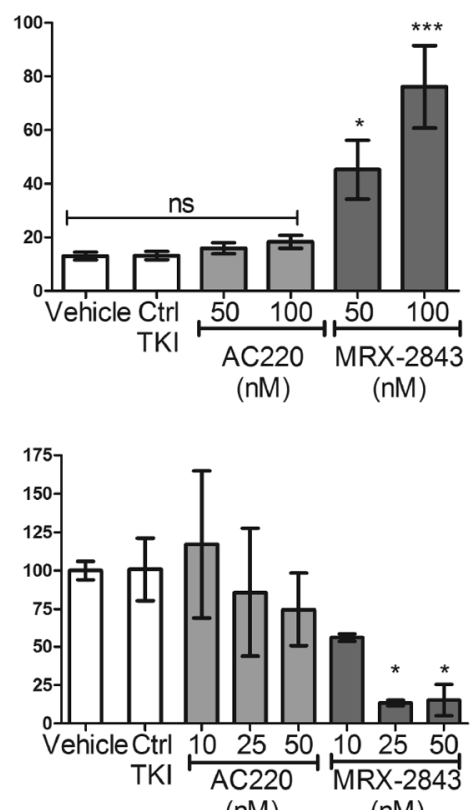

$(\mathrm{nM}) \quad(\mathrm{nM})$

Figure 7. MRX-2843 mediates functional antitumor effects in quizartinib-resistant FLT3-ITD cell lines. (A and B) Parental MOLM-14 cells and derivatives harboring mutations conferring quizartinib resistance (D835Y and F691L) were treated with MRX-2843, quizartinib (AC220), vehicle (DMSO), or a nontargeting control TKI, and apoptotic and dead cell numbers (A) and colony formation (B) were determined as described in Figure 2. Mean values and standard errors were derived from 3 independent experiments and are shown relative to vehicle-treated cells. ${ }^{*} P<0.05,{ }^{* *} P<0.01$, and ${ }^{* * *} P<0.001$, by 1 -way ANOVA. Ctrl TKI, control tyrosine kinase inhibitor; FLT3, FMS-like tyrosine kinase 3; ITD, internal tandem duplication.

rived myeloblasts, primary apheresis samples were obtained from 4 adult patients with AML at diagnosis. All of these patient samples expressed MERTK protein $\left(\mathrm{MERTK}^{+}\right)$, consistent with our previously reported findings demonstrating MERTK expression in $80 \%$ to $90 \%$ of pediatric and adult AMLs (13). In addition, 2 samples had FLT3-ITD mutations. Mononuclear cells were isolated and cultured in methylcellulose medium containing MRX-2843 or vehicle for 10 days before colonies were counted. Primary human MERTK-expressing leukemic blasts with and without FLT3-ITD mutations were very sensitive to treatment with MRX-2843, with decreased colony formation evident at concentrations as low as $5 \mathrm{nM}$ and near-complete abrogation of colony formation in response to treatment with $50 \mathrm{nM}$ MRX-2843 in 3 of the 4 samples (Figure 4, A and B). In contrast, treatment with MRX-2843 at doses up to $500 \mathrm{nM}$ had no significant effect on colony-forming potential in methylcellulose cultures of normal hematopoietic cells isolated from human cord blood, indicating a substantial therapeutic window (Figure 4C). In addition, MRX-2843 retained activity in the presence of human plasma, with a minimal shift in the dose needed to inhibit FLT3 activation in a plasma inhibitory assay (ref. 21 and Supplemental Figure 1), suggesting that clinical activity would not be significantly compromised by plasma protein binding.

While cell line-based xenograft models have been used successfully for preclinical evaluation of therapeutic agents, establishment in culture may select for specific characteristics not representative of the spectrum of biology exhibited in patients. For this reason, patient-derived xenograft models may more accurately recapitulate the biology of leukemia in humans. Therefore, orthotopic xenografts were generated from primary (Figure 5, A and B) or xenograft-passaged (Figure 5, C and D) samples from patients with AML by injection into the tail veins of NSGS mice. Peripheral disease burden was monitored by flow cytometry, and daily treatment was initiated when leukemic blasts (human $\mathrm{CD} 45^{+}\left[\mathrm{hCD} 45^{+}\right]$) were detected. Mice transplanted with a MERTK ${ }^{+}$FLT3-WT sample were used to determine the effects mediated by MERTK inhibition. In this study, once-daily treatment with $75 \mathrm{mg} / \mathrm{kg}$ MRX-2843 or an equivalent volume of vehicle was initiated 53 days after transplantation, when the average peripheral disease burden was $2.9 \% \pm 0.3 \%$. Treatment with MRX-2843 prolonged median survival from 28.5 days after treatment in vehicle-treated controls to 42 days after treatment $(P<0.01$, Figure 5A). Additionally, MRX-2843 resulted in a significant decrease in peripheral disease burden 
A

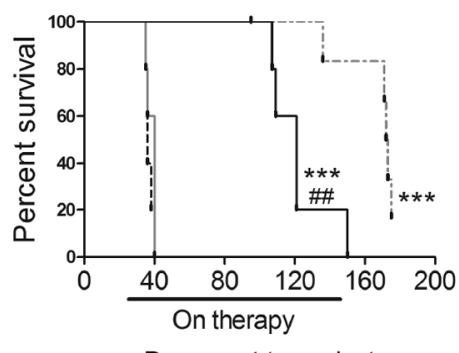

Days post-transplant

C

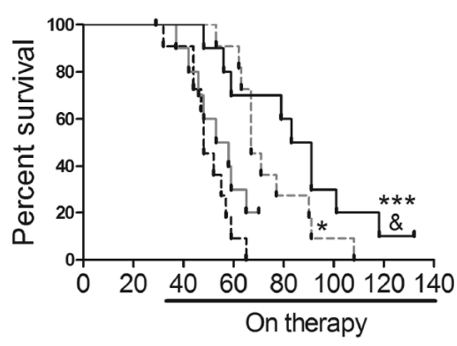

Days post-transplant
B MOLM-14:D835Y

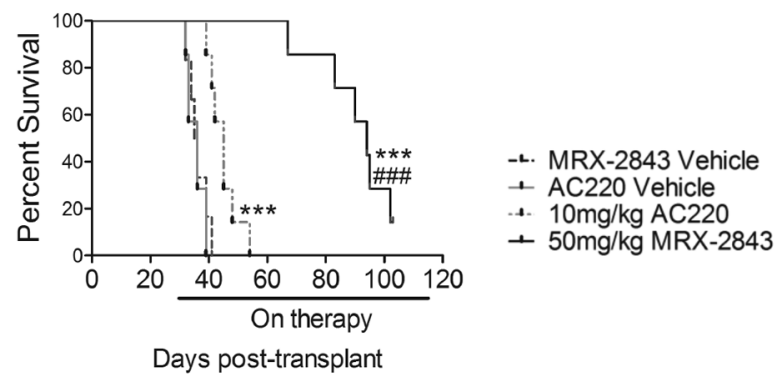

D

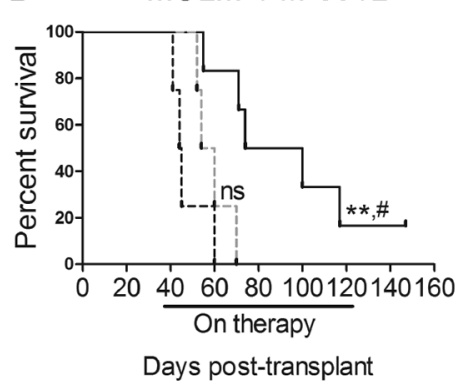

$* * * p<0.001$ versus vehicle

** $p<0.01$ versus vehicle

* $p<0.05$ versus vehicle

\#\#\# $\mathrm{p}<0.001$ versus $A C 220$

\# $p<0.01$ versus $A C 220$

\# $p<0.05$ versus $A C 220$

\& $p=n s$ versus $A C 220$

Figure 8. MRX-2843 mediates functional therapeutic effects in quizartinib-resistant FLT3-ITD xenograft models. (A-D) Kaplan-Meier curves showing survival of NSG (A, B, and D) or NSCS (C) mice with orthotopic xenografts of MOLM-14 parental (A), MOLM-14:D835Y (B), or MOLM-14:F691L (C and D) cells, treated with MRX-2843, quizartinib (AC220), MRX-2843 vehicle (saline), or quizartinib vehicle (CD). (A) Disease burden was monitored in cohorts of mice, and treatment was initiated on day 22 after transplantation, when $7.2 \% \pm 1.0 \%$ blasts were detected in the bone marrow. Treatment continued until day 145 after transplantation. Median survival was 121 days and 172.5 days for mice treated with MRX-2843 or quizartinib, respectively, compared with 36 days and 40 days for mice treated with MRX-2843 or quizartinib vehicle ( $n=5$ per group). (B) Disease burden was monitored in cohorts of mice, and treatment was initiated on day 26 after transplantation, when $6.8 \% \pm 1.2 \%$ blasts were detected in the bone marrow. Treatment continued until conclusion of the study. Median survival was 94 days and 45 days after transplantation for mice treated with MRX-2843 or quizartinib, respectively, compared with 35.5 days and 36 days for mice treated with MRX-2843 or quizartinib vehicle ( $n \geq 5$ per group). (C) Treatment was initiated on day 28 after transplantation and continued until the conclusion of the study. Median survival was 87 days and 67 days after transplantation for mice treated with MRX-2843 or quizartinib, respectively, compared with 48 days and 55.5 days for mice treated with MRX-2843 or quizartinib vehicle ( $n \geq 8$ per group). (D) Disease burden was monitored in cohorts of mice, and treatment was initiated on day 38 after transplantation, when $49 \% \pm 11 \%$ blasts were detected in the bone marrow. Treatment continued until 120 days after transplantation. Median survival was 87 days and 57 days for mice treated with MRX-2843 or quizartinib, respectively, compared with 44.5 days for mice treated with saline vehicle ( $n \geq 4$ per group). All $P$ values in A-D were determined by log-rank test. CD, hydroxybutenyl- $\beta$-cyclodextrin; FLT3, FMS-like tyrosine kinase 3; ITD, internal tandem duplication; NSG, NOD-SCID- $\gamma$; NSCS, NOD-SCID- $\gamma$ mice expressing Tg human cytokines.

relative to that seen in vehicle-treated mice $(P<0.001$, Figure 5B). A similar model was developed using a MERTK ${ }^{+}$FLT3-ITD patient's sample. Because FLT3-ITD cell lines and patient samples were generally more sensitive to MRX-2843 in cell culture assays, lower doses of $30 \mathrm{mg} / \mathrm{kg}$ and $50 \mathrm{mg} / \mathrm{kg}$ MRX-2843 were used for treatment in this study. Once-daily treatment with MRX-2843 was initiated 42 days after transplantation, with an average peripheral disease burden of $5.0 \% \pm 0.5 \%$, and continued for 48 days. Median survival was significantly increased from 23 days after treatment in the control group to 68 and 89 days in mice treated with $30 \mathrm{mg} / \mathrm{kg}$ or $50 \mathrm{mg} / \mathrm{kg} \mathrm{MRX}-2843$, respectively $(P<0.001$, Figure $5 \mathrm{C})$. As before, treatment with either dose of MRX-2843 resulted in significantly decreased peripheral disease burden relative to that seen with treatment with vehicle $(P<0.001$, Figure 5D). Interestingly, although there was no significant difference in the percentage of peripheral blasts in mice treated with $30 \mathrm{mg} / \mathrm{kg}$ or $50 \mathrm{mg} / \mathrm{kg}$ MRX-2843 on the last day of treatment (day 48), the time to disease progression was significantly prolonged in mice treated with $50 \mathrm{mg} / \mathrm{kg}$ relative to that for mice treated with the lower dose $(P<0.05$, Figure 5C).

$M R X-2843$ retains activity against quizartinib-resistant FLT3-mutant proteins. Several approaches were used to determine whether MRX-2843 has activity against FLT3-ITD-mutant proteins that are resistant to previously described FLT3 TKIs (10). First, BA/F3 cell lines stably expressing FLT3-ITD or FLT3-ITD with a resistance-conferring mutation were generated, and the impact of treatment with MRX-2843 on clonal expansion was assessed in the absence of IL-3 (8). In this system, cytokine-independent expansion is driven by FLT3-ITD activity. An $\mathrm{IC}_{50}$ of $3.0 \pm 0.5 \mathrm{nM}$ MRX-2843 was observed in BA/F3 cells expressing FLT3- 
ITD alone, indicating potent inhibition of FLT3-ITD (Figure 6A and Supplemental Table 2). MRX-2843 retained inhibitory activity against clinically relevant point mutations at the D835 and F691 loci, with $\mathrm{IC}_{50}$ values of $7.2 \pm 1.3 \mathrm{nM}$ and $20.4 \pm 4.3 \mathrm{nM}$ in BA/F3 lines expressing FLT3-ITD D835Y and FLT3-ITD F691L, respectively (Figure 6A and Supplemental Table 2). In contrast, point mutations at these loci rendered resistance to the FLT3 inhibitor quizartinib (AC220; Supplemental Figure 3). MRX-2843 retained activity against additional FLT3-ITD D835, F691, and Y842 locus mutants that were identified using a mutagenesis screen to determine changes causing resistance to quizartinib (ref. 8, Supplemental Figure 3, and Supplemental Table 2). Two derivatives of the human FLT3-ITD AML cell line MOLM-14 that acquired either a D835Y or F691L mutation after selection in escalating doses of quizartinib (11) were used to confirm and extend these findings. In this model, treatment with MRX-2843 decreased expansion of the parental MOLM-14 cells with an $\mathrm{IC}_{50}$ of $19.1 \pm 4.7 \mathrm{nM}$ and demonstrated minimally reduced activity against both MOLM-14:D835Y and MOLM-14:F691L derivative cells with $\mathrm{IC}_{50}$ values of $21.7 \pm 4.3$ $\mathrm{nM}$ and $34.8 \pm 7.9 \mathrm{nM}$, respectively (Figure 6B and Supplemental Table 2). In biochemical assays, FLT3 phosphorylation and downstream signaling through STAT5, AKT, and ERK1/2 were abrogated in the MOLM-14 parental cell line treated with either quizartinib or MRX-2843 at similar doses of 10 to $25 \mathrm{nM}$ (Figure 6C and Supplemental Figure 2). In MOLM-14:D835Y and MOLM-14:F691L cells, MRX-2843 retained potent activity with similar (D835Y) or slightly increased (F691L) doses mediating inhibition of FLT3 and downstream signaling. In contrast, significantly higher doses of up to $100 \mathrm{nM}$ quizartinib were required to affect the activation of these pathways, particularly in the F691L cell line. To better understand the ability of MRX-2843 to retain activity against aa substitutions at these loci, a structural model of the MRX-2843:FLT3 complex was developed. The binding mode of MRX-2843 to FLT3 was derived from a crystal structure featuring UNC569, a close MRX-2843 analog, bound to MERTK (PDB:3TCP; ref. 22). The overlaid MRX-2843 and quizartinib bound to F691L mutant and WT FLT3, respectively, are shown in Figure 6D. The binding model clearly suggests that MRX-2843 and quizartinib adopt substantially different inhibition mechanisms. In particular, quizartinib features a "tail" (including the isoxazole group) that penetrates into the back pocket formed by the c-helix and the activation loop. Consequently, it can only bind the so-called "DFG-out," catalytically inactive, conformation and is therefore a type II kinase inhibitor (23). In contrast, the bound MRX-2843 is confined within the adenine-binding pocket, such that it has equal potencies with respect to both the "DFG-in" and "DFG-out" enzyme conformations and is therefore a type I kinase inhibitor. This binding mode makes MRX-2843 much more active against the F691L FLT3-mutant protein than quizartinib. For instance, the flexible cyclopropylethyl group in MRX-2843 forms favorable van der Waals interactions equally well with either the leucine or phenylalanine side chains, while quizartinib binding is dependent on a stacking interaction with the F691 side chain. Moreover, phenylalanine is also likely to favor a metastable conformation required for quizartinib engagement (24).

$M R X-2843$ mediates functional antileukemic effects in quizartinib-resistant FLT3-ITD cell lines. Comparison of MRX-2843 and quizartinib in apoptosis and colony-forming assays was used to determine the functional effects of MRX-2843 in quizartinib-resistant cell lines. As expected, the parental MOLM-14 cell line was sensitive to both MRX-2843 and quizartinib at similar doses (Figure 7, A and B). Specifically, treatment with $50 \mathrm{nM}$ MRX-2843 or quizartinib induced cell death in $78.8 \% \pm 12.3 \%$ or $91.5 \% \pm 3.8 \%$ of cells, respectively, and a dose of $10 \mathrm{nM}$ inhibited colony formation by $48.1 \% \pm 20.3 \%$ or $98.3 \% \pm 0.7 \%$. As predicted based on the biochemical assays, MRX-2843 retained the ability to induce apoptosis (Figure 7A) and inhibit colony formation (Figure 7B) in both the MOLM-14:D835Y and MOLM-14:F691L mutant cell lines, with similar (D835Y) or slightly reduced (F691L) potency relative to that of parental MOLM-14 cells. In contrast, quizartinib did not significantly induce apoptosis or alter colony formation in MOLM14:D835Y or MOLM-14:F691L cell lines.

MRX-2843 prolongs survival in quizartinib-resistant FLT3-ITD xenograft models. To determine the therapeutic effects mediated by MRX-2843 in quizartinib-resistant FLT3-ITD AML models, the MOLM-14 parental and derivative cell lines were used to generate orthotopic xenografts in NSG mice, and the impact of treatment with MRX-2843 or quizartinib was determined. Mice were treated with $50 \mathrm{mg} / \mathrm{kg} \mathrm{MRX-}$ $2843,10 \mathrm{mg} / \mathrm{kg}$ quizartinib (the maximum published dose; refs. 25, 26), or an equivalent volume of vehicle (saline or $50 \mathrm{mg} / \mathrm{kg}$ hydroxybutenyl- $\beta$-cyclodextrin in saline, respectively) administered once daily by oral gavage. In MOLM-14 parental xenografts, both quizartinib and MRX-2843 increased median survival compared with that of vehicle-treated mice (172.5 days versus 40 days and 121 days versus 36 days, respectively, $P<0.001$, Figure $8 \mathrm{~A})$. In this model, quizartinib was more effective than MRX-2843 $(P<0.005)$, 
although higher doses of MRX-2843 were not evaluated. In MOLM-14:D835Y xenografts, quizartinib prolonged survival compared with that of vehicle-treated mice, but the effect was minimal (median survival 45 days vs. 36 days, $P<0.001$ ). In contrast, MRX-2843 resulted in a greater than 2-fold increase in median survival in MOLM-14:D835Y xenografts (median survival 94 days vs. 35.5 days, $P<0.001$; Figure $8 \mathrm{~B}$ ). In MOLM-14:F691L xenografts, treatment with MRX-2843 prolonged survival by almost 2-fold in NSG (Figure 8C) and NSGS (Figure 8D) mice (median survival 87 vs. 44.5 days and 87 vs. 48 days, respectively, $P<$ 0.005). Treatment with quizartinib also increased median survival in NSG (57 vs. 44.5 days) and NSGS (67 vs. 55.5 days, $P<0.05)$ mice, although this difference was not significant in NSG mice. Similarly, increased survival was observed in response to treatment with MRX-2843 versus quizartinib, but the difference was only significant in NSG mice.

\section{Discussion}

Here, we describe the preclinical characterization of MRX-2843, a small-molecule TKI of MERTK and FLT3, 2 attractive targets in AML. MRX-2843 inhibits phosphorylation of MERTK and FLT3 and activation of their downstream effectors, leading to induction of apoptosis and decreased clonal expansion and/ or colony formation in AML cell lines and patient samples. In addition, we demonstrate that daily oral treatment with MRX-2843 prolongs survival in cell line- and patient-derived orthotopic mouse xenograft models of MERTK-positive, FLT3-ITD-positive, and dual-positive AML. The effects of treatment with MRX-2843 in MERTK-dependent models recapitulate the phenotypes observed in response to shRNAmediated MERTK inhibition, providing evidence that MRX-2843 antileukemic activity is a consequence of MERTK inhibition in this context (13). Similarly, treatment with MRX-2843 mediates antileukemic activity in FLT3-dependent models with a potency similar to that of quizartinib. MRX-2843 retains activity against FLT3 proteins, with aa changes at D835 or F691, two loci where mutations have been identified in patients resistant to treatment with other FLT3 inhibitors. Taken together, these data validate MRX-2843 for translational application as a dual MERTK and FLT3 inhibitor. Given the near-ubiquitous expression of MERTK and the $20 \%-30 \%$ incidence of activating mutations in FLT3, MRX-2843 could be a widely applicable therapeutic in patients with AML.

In addition, MRX-2843 is an ATP-competitive type 1 inhibitor, and, unlike quizartinib and other type 2 inhibitors that bind target proteins in their inactive conformation (24), acquisition of mutations that stabilize the active form of the target protein is unlikely to be a mechanism of acquired resistance. Indeed, MRX-2843 retains activity against FLT3-mutant proteins containing activating aa changes at D835.

Furthermore, MRX-2843 retains activity against both of the clinically relevant FLT3 mutants that have been identified in patients resistant to therapy with FLT3 TKIs. Point mutations at 2 loci, D835 (activation loop) and F691 (gatekeeper), emerge in patients who relapse while on therapy with quizartinib, and approximately half of the recurrent tumors contain polyclonal mutations (8). Mutations at both loci confer significant cross-resistance to sorafenib in vitro, and acquisition of a D835Y point mutation has been detected in patients who relapsed while receiving sorafenib therapy (9). Crenolanib is a FLT3 inhibitor with activity against the D835-mutant protein and is currently in clinical trials for treatment of AML with FLT3-activating mutations (10, 27). However, gatekeeper mutations render relative resistance to crenolanib, such that patients remain susceptible to the development of resistance via acquisition of mutations at the F691 loci or selection of existing F691-mutant clones. This is of particular concern, given the high number of patients who develop polyclonal FLT3 mutations. Similarly, the multi-kinase inhibitor ponatinib has activity in FLT3-ITD AMLs with F691L mutation, but is not active against activation loop mutants (11). MRX-2843 is a FLT3 TKI that retains activity against both D835Y- and F691L-mutant proteins and may thereby provide a therapeutic option for patients with tumors that have acquired point mutations mediating resistance to current FLT3 inhibitors without the potential to select additional mutations. Clearly, the emergence of point mutations in FLT3 is not the only mechanism of resistance in FLT3-ITD AMLs, but it appears to be the most common one (28). A similar mechanism mediates resistance to TKIs targeting BCR-ABL in chronic myeloid leukemia (CML) (29) and acute lymphocytic leukemia (30), indicating the importance of this mechanism. While treatment with MRX-2843 was sufficient to delay leukemia progression and doubled or even tripled survival in multiple xenograft models of $A M L$, including patient-derived xenografts, all of the mice eventually succumbed to disease. The reason for the lack of curative growth suppression in MRX-2843treated models remains to be elucidated. 
Several lines of evidence suggest that dual inhibition of MERTK and FLT3 will provide greater therapeutic benefit than inhibition of either target alone. MERTK signaling has a variety of functional consequences in cancer cells, but its ability to promote tumor cell survival is particularly important (15). For instance, in acute lymphoblastic leukemia models, stimulation with GAS6, a MERTK ligand, promotes chemoresistance (31), and shRNA-mediated MERTK knockdown enhances sensitivity to cytotoxic chemotherapeutics $(12,14)$. Studies investigating the role of MERTK in resistance to targeted therapeutics, such as those directed at FLT3, have not been reported. However, another member of the TAM family, AXL, has been implicated in acquired resistance to a variety of TKIs and in multiple tumor types. Notably, in AML cell lines, AXL knockdown via shRNA restored sensitivity to the FLT3 inhibitor midostaurin (32). Given the known role of MERTK in promoting survival and resistance to traditional chemotherapies and its close relationship to AXL, it is likely that MERTK also has a role in mediating resistance to targeted therapies, and thus dual inhibition of MERTK and FLT3 may decrease the development of resistance relative to inhibition of FLT3 alone.

In addition to delaying or preventing development of resistance, coinhibition of MERTK and FLT3 may improve therapeutic efficacy by other mechanisms. Overexpression of the TAM family kinase ligand GAS6 is a poor prognostic indicator in cytogenetically normal AML, and depletion of GAS6 results in decreased FLT3 phosphorylation and clonal expansion of FLT3-ITD cell lines and primary patient samples $(33,34)$. While this effect is mediated, at least in part, by inhibition of AXL, introduction of AXL siRNA only partially reduced FLT3 phosphorylation, and depletion of GAS6 had a much more substantial impact, suggesting that MERTK and/or TYRO-3 activity also play a role in promoting FLT3 activity.

Enzymatic assays reveal very close $\mathrm{IC}_{50}$ values of MRX-2843 for MERTK (1.3 nM) and FLT3 (0.64 $\mathrm{nM}$ ). The heightened cell-based assay potency of MRX-2843 in cell lines expressing FLT3-ITD is approximately 3- to 4-fold greater than that in MERTK ${ }^{+}$FLT3-WT cells. This may simply reflect unique cell line attributes, but it may also reflect the presumed action of FLT3-ITD and MERTK, with the former being a classic oncogenic driver and the latter being a survival factor providing nononcogenic addiction.

One goal of targeted therapies with selectivity for tumor cells is to minimize toxicities. Daily treatment with MRX-2843 was well tolerated in mice for up to 120 days. In addition, MRX-2843 had a 10-fold differential effect on myeloblasts derived from patients with $\mathrm{AML}$ and normal mononuclear cells derived from human cord blood samples, indicating a large therapeutic window. The primary physiologic roles of MERTK include immune regulation and clearance of apoptotic debris (15). Complete loss of function of MERTK protein has been identified in humans, with the primary clinical effect of retinitis pigmentosa, likely due to defects in apoptotic clearance in the retina (35). In a rat model, the phenotype was reversible with exogenous MERTK expression (36), indicating that short-term pharmacologic inhibition of MERTK is unlikely to lead to permanent adverse effects. With regard to FLT3 inhibition, the effects of inhibitors such as quizartinib have been well described, with myelosuppression being one of the most prominent toxicities, likely due to the combined inhibition of both FLT3 and c-KIT $(37,38)$. In comparison with current FLT3 inhibitors, MRX-2843 has reduced activity against c-KIT, and we do not anticipate combined inhibition of FLT3 with MERTK to exacerbate myelosuppression, as MERTK is not expressed on normal hematopoietic cells (14).

In summary, MRX-2843 is a potent small-molecule inhibitor of both MERTK and FLT3 that has antileukemic activity in preclinical assays and robust therapeutic activity in vivo. MRX-2843 is selective for leukemia cells compared with normal hematopoietic progenitors, suggesting a wide therapeutic window. Moreover, MRX-2843 is active against FLT3 proteins containing clinically relevant activation loop or gatekeeper mutations and is one of the only FLT3 TKIs reported to effectively target both mutant proteins. These observations suggest that treatment with MRX-2843 will provide more durable responses compared with those elicited by current FLT3 inhibitors and indicate the potential utility of MRX-2843 for the treatment of patients with acquired resistance to these agents. In addition, the high frequency of FLT3-ITD mutations and nearly ubiquitous overexpression of MERTK in AML make dual targeting a particularly attractive therapeutic strategy. Together, these observations validate MRX-2843 as a translational agent and support its clinical development for the treatment of AML.

\section{Methods}

Inhibitors. MRX-2843 and the control TKI were synthesized as previously described (20). The amount of kinase inhibitor required for $90 \%$ inhibition in vivo was estimated using Michaelis-Menton kinetic equations as previously described (20). Quizartinib (AC220) was obtained from the Colorado Clinical and 
Translational Sciences Institute Medicinal Chemistry core facility as a 1:4 formulation with hydroxybutenyl- $\beta$-cyclodextrin (CD). For in vitro studies, stock solutions were prepared in DMSO (Sigma-Aldrich), and the DMSO vehicle control concentration was equivalent to the highest dose of test agent for the experiment. For in vivo studies, test agents were either dissolved (MRX-2843) or prepared in a homogeneous suspension (quizartinib and CD) in saline.

Patient samples and cell culture. Deidentified cord blood from healthy donors and peripheral blood apheresis samples from patients with AML at the time of diagnosis were obtained from the University of Colorado. Samples from patients with AML were cultured as previously described (39).

The cell lines MV4-11 and Kasumi-1 were purchased from the American Type Culture Collection (ATCC) in 2008 and last authenticated in June 2013 and April 2014, respectively. NOMO-1 was obtained from the DSMZ (German Collection of Microorganisms and Cell Culture) in 2006 and last authenticated in April 2014. MOLM-14 cells were obtained from the laboratory of Scott Kogan (Department of Laboratory Medicine, UCSF, San Francisco, California, USA) in 2008 and authenticated in November 2013. Cell-line identities were confirmed using short tandem repeat microsatellite loci analysis. Frozen stocks of all cell lines were generated at the time of authentication, and stocks were thawed for experiments no more than 3 months before use. MOLM-14:D835Y, MOLM-14:F691L, and Ba/F3 cells expressing FLT3ITD were previously described $(8,11)$. All cell lines were cultured in RPMI medium (HyClone) supplemented with 10\% FBS (or 20\% for Kasumi-1) and penicillin-streptomycin (complete RPMI [cRMPI]). All cell lines were free of Mycoplasma.

Immunoblot analysis of downstream signaling pathways. Three million exponentially growing cells were cultured in CRPMI supplemented with vehicle, control TKI, or the indicated concentration of kinase inhibitor for 1.5 hours. Cell lysates were prepared in lysis buffer (50 mM HEPES, pH 7.5, $150 \mathrm{mM} \mathrm{NaCl}, 10 \mathrm{nM}$ EDTA, 10\% glycerol, 1\% Triton X-100) supplemented with protease and phosphatase inhibitors. Proteins were quantitated, resolved on $8 \%$ Tris-Glycine SDS-PAGE gels (Invitrogen), and transferred onto nitrocellulose membranes. Membranes were blocked with 5\% milk in tris-buffered saline with $0.1 \%$ Tween-20 and then incubated with Abs specific to the proteins of interest. Proteins were visualized on x-ray film using HRP for chemiluminescence detection (PerkinElmer). Phosphorylated proteins were first detected, then membranes were stripped and probed for total protein or actin, which was used as a protein concentration control. Anti-actin Ab (sc-1616) and donkey anti-goat IgG-HRP (sc-2020) were from Santa Cruz Biotechnology Inc. Abs against phosphorylated STAT5 (p-STAT5) (Tyr694, \#9359); p-STAT6 (Tyr641, \#9364); p-AKT (Ser473, \#9271L); p-ERK1/2 (Thr202/Tyr204, \#9106); STAT5 (\#9358); STAT6 (\#9362); AKT (\#9272); and ERK1/2 (\#9102) were from Cell Signaling Technology. Goat anti-mouse IgG-HRP and goat anti-rabbit IgG-HRP were from Bio-Rad. Proteins were quantitated by densitometry using ImageJ software (NIH). The data shown are representative of at least 3 independent experiments.

Immunoblot analysis of FLT3 and MERTK. For analysis of p-FLT3 and p-MERTK only, cells were treated as above, but $0.12 \mathrm{mM}$ pervanadate (an irreversible phosphatase inhibitor) was added to the cultures for 3 minutes (FLT3) or 10 minutes (MERTK) prior to preparation of the cell lysates to stabilize the phosphorylated proteins. Lysates were incubated with anti-MERTK (MAB8912; R\&D Systems) or anti-FLT3 S18 (sc-480; Santa Cruz Biotechnology Inc.) Abs overnight on a rotator, and recombinant protein G sepharose beads (Invitrogen) were added for an additional 2 hours. Beads were washed 3 times with lysis buffer, and bound proteins were eluted by boiling in $2 \times$ Laemmli sample buffer (Bio-Rad). Proteins were detected by immunoblotting as described above using p-MERTK (Y749, Y753, Y754; Phosphosolutions), p-FLT3 (\#3461; Cell Signaling Technology), MERTK (ab52968; AbCam), or FLT3 (sc-480; Santa Cruz Biotechnology Inc.) Abs. Data shown are representative of at least 3 independent experiments.

Clonal expansion assay. Exponentially growing cells (BA/F3: $5 \times 10^{3}$ cells/well; others: $1 \times 10^{4}$ cells/ well) were cultured in triplicate for 48 hours in $0.1 \mathrm{ml} \mathrm{cRPMI}$ containing the appropriate concentration of test agent or vehicle. ATP levels were determined as an indicator of viable cell numbers using CellTiterGlo reagent (Promega) according to the manufacturer's recommendation. Luminescence was detected and quantitated using a SpectraMax M3 microplate reader and SpectraMax Pro Software (Molecular Devices). Luminescence intensities relative to the mean intensity in vehicle-treated samples were calculated, and $\mathrm{IC}_{50}$ values were determined by nonlinear best-fit regression analysis using Prism 5 software, version 5.01 (GraphPad Software).

Apoptosis assay. Cells $\left(3 \times 10^{5}\right.$ per sample) were cultured in cRPMI with vehicle, control TKI, or kinase inhibitor at the indicated concentration. After 72 hours, cells were harvested and resuspended in PBS with 
$1 \mu \mathrm{M}$ YO-PRO-1 iodide (Invitrogen) and $1.5 \mu \mathrm{M}$ propidium iodide (Invitrogen) for 15 to 30 minutes prior to assessment of dye uptake by flow cytometry using an FC500 cytometer and CXP analysis software (both from Beckman Coulter).

Colony-forming assays. Cell lines were cultured (10,000 cells/sample) in $0.35 \%$ Noble agar on a $0.5 \%$ Noble agar base layer and overlaid with CRPMI containing kinase inhibitor or vehicle. The overlying medium was replaced 2-3 times per week, and vehicle treatment was assessed in duplicate. After 14 days or 21 days (Kasumi-1 cells only), colonies were stained with $1 \mathrm{mg} / \mathrm{ml}$ nitrotetrazolium blue (Sigma-Aldrich) for 4 hours and counted using a GelCount colony counter (Oxford Optronix). Mononuclear cells were isolated from human cord blood and samples from AML patients using Ficoll-Paque PLUS (GE Healthcare Life Sciences). Patient samples were cultured in triplicate at a density of $1 \times 10^{6}$ cells/ml in MethoCult H4434 Classic Methylcellulose-Based Medium with Recombinant Cytokines for Human Cells (STEMCELL Technologies) containing MRX-2843 or vehicle. Colonies were counted after 10 days using the GelCount colony counter (Oxford Optronix). Cord blood cells were incubated for 1 hour in serum-free Iscove's modified Dulbecco's medium (IMDM) (HyClone) supplemented with BIT 9500 Serum Substitute (STEMCELL Technologies), low-density lipoproteins (EMD Millipore), and 2-ME (Sigma-Aldrich), and then cultured in triplicate at a density of $2 \times 10^{6}$ cells $/ \mathrm{ml}$ in Methocult H4434 methylcellulose containing MRX-2843 or vehicle. Colonies were manually counted in a blinded manner after 14 days.

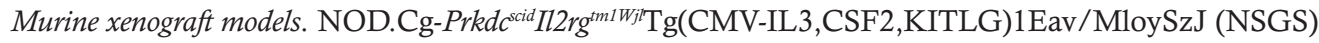
mice and NOD.Cg-Prkdc sid $I l 2 r g^{t m 1 W_{j 1}} / \mathrm{SzJ}$ (NSG) mice were purchased from The Jackson Laboratory or bred in-house and maintained under sterile conditions. Established leukemia cell lines or mononuclear cells isolated from samples from patients with $\mathrm{AML}\left(1 \times 10^{6}\right.$ to $2.5 \times 10^{6}$ per mouse $)$ were suspended in PBS and injected into the tail veins of NSG or NSGS mice to establish xenografts. All mice were 4-6 months of age at the time of injection and were male, with the exception of the NOMO-1, MOLM14:D835Y, and MOLM-14:F691L NSG xenografts, which were established in female mice. Myeloblasts were detected in peripheral blood (patient-derived xenografts) or bone marrow (MOLM-14 xenografts) samples after staining with a FITC-conjugated anti-human CD45 Ab (BD Biosciences). Samples were analyzed by flow cytometry using a Gallios flow cytometer and Kaluza software (both from Beckman Coulter). After engraftment, the mice were weighed and treated once daily with MRX-2843, quizartinib, or vehicle administered by oral gavage in a volume of $10 \mathrm{ml} / \mathrm{kg}$. When mice appeared ill or lost more than $20 \%$ of their body weight, they were euthanized.

Structural modeling. A high-resolution crystal structure of the MERTK protein kinase domain in complex with UNC569 (PDB:3TCP; ref 22) was used as a structural template for modeling the MRX -2843:FLT3 complex. To this end, the 3TCP MERTK kinase structure was aligned with the 4XUF FLT3 structure and UNC569 was transferred into 4XUF and "alchemically" transformed into MRX-2843. All modeling and calculations were performed using the Maestro 10.3 Software Suite (Schrödinger). The resulting MRX-2843:FLT3 complex was refined in implicit solvent by means of Monte Carlo sampling and the OPLS-2005 force field using the Prime module. All protein structures were preprocessed using the protein preparation workflow available in Maestro.

Statistics. Data were analyzed by 1-way ANOVA with Bonferroni's test for multiple comparisons. Data are presented as the mean \pm SEM. $P$ values of less than 0.05 were considered statistically significant. $\mathrm{IC}_{50}$ values were determined by nonlinear best-fit regression analysis. Kaplan-Meier survival curves were compared using the log-rank test. All statistical analyses were performed using Prism 5 software, version 5.01 (GraphPad Software).

Study approval. All animal experiments were conducted in accordance with the relevant regulatory standards set forth and were approved by the IACUC of the University of Colorado. Patient samples were obtained from the University of Colorado after written informed consent was received, in accordance with the principles of the Declaration of Helsinki. All experiments conformed to the regulatory standards approved by the Colorado Multiple Institutional Review Board.

\section{Author contributions}

KAM, CCS, DD, CL, ABLS, MGH, EAL, GDK, MAS, WZ, DK, and XW designed and performed the experiments and analyzed the data. CTJ, SVF, HSE, NPS, and DKG analyzed the data. KAM, CCS, DD, ABLS, DK, HSE, and DKG wrote the manuscript. 


\section{Acknowledgments}

The authors thank the University of Colorado Cancer Center Flow Cytometry Core for technical assistance (P30CA046934); the University of Colorado Diabetes and Endocrinology Research Center Molecular Biology Core Facility (NIH P30DK57516) for cell line authentication services; and Lauren S. Page for her technical assistance. This work was supported by grants from the NIH (2T32CA082086-11, to K.A. Minson and 1R01CA137078, to D.K. Graham); the Leukemia and Lymphoma Society Translation Research Program (to D.K. Graham); the University of North Carolina Cancer Research Fund; and federal funds from the National Cancer Institute's Experimental Therapeutics (NExT) program, NIH, under contract HHSN261200800001E. C.C. Smith is an American Society of Hematology Faculty Scholar. C. Libbrecht received funding from L'Institut Servier.

Address correspondence to: Douglas K. Graham, Department of Pediatrics, Section of Hematology, Oncology and Bone Marrow Transplantation, Emory University School of Medicine, 2015 Uppergate Drive NE, $4^{\text {th }}$ Floor, Atlanta, Georgia 30322, USA. Phone: 404.785.3874; E-mail: douglas.graham@choa.org.

1. Redaniel MT, Pulte D, Jeffreys M. Survival disparities by age and country of diagnosis for patients with acute leukemia. Leuk Lymphoma. 2015;56(10):2787-2792.

2. Karol SE, et al. Prognostic factors in children with acute myeloid leukaemia and excellent response to remission induction therapy. Br J Haematol. 2015;168(1):94-101.

3. Pollyea DA, Kohrt HE, Medeiros BC. Acute myeloid leukaemia in the elderly: a review. Br J Haematol. 2011;152(5):524-542.

4. Leung W, Hudson MM, Strickland DK, Phipps S, Srivastava DK, Ribeiro RC. Late effects of treatment in survivors of childhood acute myeloid leukemia. J Clin Oncol. 2000;18(18):3273-3279.

5. Kottaridis PD, et al. The presence of a FLT3 internal tandem duplication in patients with acute myeloid leukemia (AML) add important prognostic information to cytogenetic risk group and response to the first cycle of chemotherapy: analysis of 854 patients from the United Kingdom Medical Research Council AML 10 and 12 trials. Blood. 2001;98(6):1752-1759.

6. Meshinchi S, et al. Prevalence and prognostic significance of Flt3 internal tandem duplication in pediatric acute myeloid leukemia. Blood. 2001;97(1):89-94.

7. Levis MJ, et al. Final Results of a Phase 2 Open-Label, Monotherapy Efficacy and Safety Study of Quizartinib (AC220) in Patients with FLT3 ITD Positive or Negative Relapsed/Refractory Acute Myeloid Leukemia After Second-Line Chemotherapy or Hematopoietic Stem Cell Transplantation. Presented at: 54th ASH Annual Meeting and Exposition; December 8-11, 2012; Atlanta, Georgia, USA. Session 615.

8. Smith CC, et al. Validation of ITD mutations in FLT3 as a therapeutic target in human acute myeloid leukaemia. Nature. 2012;485(7397):260-263.

9. Man $\mathrm{CH}$, et al. Sorafenib treatment of FLT3-ITD $(+)$ acute myeloid leukemia: favorable initial outcome and mechanisms of subsequent nonresponsiveness associated with the emergence of a D835 mutation. Blood. 2012;119(22):5133-5143.

10. Smith CC, et al. Crenolanib is a selective type I pan-FLT3 inhibitor. Proc Natl Acad Sci U S A. 2014;111(14):5319-5324

11. Smith CC, et al. Activity of ponatinib against clinically-relevant AC220-resistant kinase domain mutants of FLT3-ITD. Blood. 2013;121(16):3165-3171.

12. Brandao LN, et al. Inhibition of MerTK increases chemosensitivity and decreases oncogenic potential in T-cell acute lymphoblastic leukemia. Blood Cancer J. 2013;3:e101.

13. Lee-Sherick AB, et al. Aberrant Mer receptor tyrosine kinase expression contributes to leukemogenesis in acute myeloid leukemia. Oncogene. 2013;32(46):5359-5368.

14. Linger RM, et al. Mer receptor tyrosine kinase is a therapeutic target in pre-B-cell acute lymphoblastic leukemia. Blood. 2013;122(9):1599-1609.

15. Graham DK, DeRyckere D, Davies KD, Earp HS. The TAM family: phosphatidylserine sensing receptor tyrosine kinases gone awry in cancer. Nat Rev Cancer. 2014;14(12):769-785.

16. Knubel KH, Pernu BM, Sufit A, Nelson S, Pierce AM, Keating AK. MerTK inhibition is a novel therapeutic approach for glioblastoma multiforme. Oncotarget. 2014;5(5):1338-1351.

17. Linger RM, et al. Mer or Axl receptor tyrosine kinase inhibition promotes apoptosis, blocks growth, and enhances chemosensitivity of human non-small cell lung cancer. Oncogene. 2013;32(29):3420-3431.

18. Schlegel J, et al. MERTK receptor tyrosine kinase is a therapeutic target in melanoma. J Clin Invest. 2013;123(5):2257-2267.

19. Lee-Sherick AB, et al. Efficacy of a Mer and Flt3 tyrosine kinase small molecule inhibitor, UNC1666, in acute myeloid leukemia. Oncotarget. 2015;6(9):6722-6736.

20. Zhang W, et al. UNC2025, a potent and orally bioavailable MER/FLT3 dual inhibitor. J Med Chem. 2014;57(16):7031-7041.

21. Levis M, et al. Plasma inhibitory activity (PIA): a pharmacodynamics assay reveals insights into the basis for cytotoxic response to FLT3 inhibitors. Blood. 2006;108(10):3477-3483

22. Liu J, et al. Discovery of novel small molecule mer kinase inhibitors for the treatment of pediatric acute lymphoblastic leukemia. ACS Med Chem Lett. 2012;3(2):129-134.

23. Zuccotto F, Ardini E, Casale E, Angiolini M. Through the "gatekeeper door": exploiting the active kinase conformation. JMed Chem. 2010;53(7):2681-2694.

24. Zorn JA, Wang Q, Fujimura E, Barros T, Kuriyan J. Crystal structure of the FLT3 kinase domain bound to the inhibitor Quizartinib (AC220). PLoS One. 2015;10(4): e0121177.

25. Zarrinkar PP, et al. AC220 is a uniquely potent and selective inhibitor of FLT3 for the treatment of acute myeloid leukemia 
(AML). Blood. 2009;114(14):2984-2992.

26. Taylor SJ, Dagger SA, Thien CB, Wikstrom ME, Langdon WY. Flt3 inhibitor AC220 is a potent therapy in a mouse model of myeloproliferative disease driven by enhanced wild-type Flt3 signaling. Blood. 2012;120(19):4049-4057.

27. Galanis A, et al. Crenolanib is a potent inhibitor of FLT3 with activity against resistance-conferring point mutants. Blood. 2014;123(1):94-100.

28. Daver N, et al. Secondary mutations as mediators of resistance to targeted therapy in leukemia. Blood. 2015;125(21):3236-3245.

29. Shah NP, et al. Multiple BCR-ABL kinase domain mutations confer polyclonal resistance to the tyrosine kinase inhibitor imatinib (STI571) in chronic phase and blast crisis chronic myeloid leukemia. Cancer Cell. 2002;2(2):117-125.

30. Foà R, et al. Dasatinib as first-line treatment for adult patients with Philadelphia chromosome-positive acute lymphoblastic leukemia. Blood. 2011;118(25):6521-6528.

31. Shiozawa Y, Pedersen EA, Taichman RS. GAS6/Mer axis regulates the homing and survival marrow niche. Exp Hematol. 2010;38(2):132-140

32. Park IK, et al. Receptor tyrosine kinase Axl is required for resistance of leukemic cells to FLT3-targeted therapy in acute myeloid leukemia. Leukemia. 2015;29(12):2382-2389.

33. Whitman SP, Kohlschmidt J, Maharry K, Volinia S, Mrozek K, Nicolet D. GAS6 expression identifies high-risk adult AML patients: potential implications for therapy. Leukemia. 2014;28(6):1252-1258.

34. Park IK, Mishra A, Chandler J, Whitman SP, Marcucci G, Caligiuri MA. Inhibition of the receptor tyrosine kinase Axl impedes activation of the FLT3 internal tandem duplication in human acute myeloid leukemia: implications for Axl as a potential therapeutic target. Blood. 2013;121(11):2064-2073.

35. Gal A, et al. Mutations in MERTK, the human orthologue of the RCS rat retinal dystrophy gene, cause retinitis pigmentosa. Nat Genet. 2000;26(3):270-271

36. Vollrath D, et al. Correction of the retinal dystrophy phenotype of the RCS rat by viral gene transfer of Mertk. Proc Natl Acad Sci US A. 2001;98(22):12584-12589.

37. Mackarehtschian K, Hardin JD, Moore KA, Boast S, Goff SP, Lemischka IR. Targeted disruption of the flk2/flt3 gene leads to deficiencies in primitive hematopoietic progenitors. Immunity. 1995;3(1):147-161.

38. Cortes JE, et al. Phase I study of quizartinib administered daily to patients with relapsed or refractory acute myeloid leukemia irrespective of FMS-like tyrosine kinase 3-internal tandem duplication status. J Clin Oncol. 2013;31(29):3681-3687.

39. Klco JM, et al. Genomic impact of transient low-dose decitabine treatment on primary AML cells. Blood. 2013;121(9):16331643. 